\title{
Correction to: Borage (Borago officinalis) Seed
}

\author{
Beenu Tanwar, Ankit Goyal, Vikas Kumar, Prasad Rasane, \\ and Manvesh Kumar Sihag
}

\section{Correction to:}

Chapter 14 in: B. Tanwar, A. Goyal (eds.), Oilseeds: Health Attributes and Food Applications, https://doi.org/10.1007/978-981-15-4194-0_14

The original version of the chapter was inadvertently published with an error in the affiliation.

Affiliation in chapter: Department of Food Science and Technology, College of Agriculture, Punjab Agricultural University, Ludhiana, Punjab, India.

Correct Affiliation: Department of Food Technology and Nutrition, School of Agriculture, Lovely Professional University, Phagwara, Punjab, India. Email: rasaneprasad@gmail.com

The affiliation has been corrected and approved. 